\title{
Effect of the dimension of a tone hole on the fundamental frequency of flutes
}

\author{
Kenko Ota* \\ Faculty of Electrical, Electronics and Communication Engineering, Nippon Institute of Technology, \\ 4-1 Gakuendai, Miyashiro-machi, Minamisaitama-gun, Saitama, 345-8501 Japan
}

(Received 23 October 2020, Accepted for publication 23 March 2021)

Keywords: Flute, Tone hole, Resonance frequency, Artificial blowing system

\section{Introduction}

In flute design, the size and position of tone holes are important parameters that affect the fundamental frequency of the flute and the ease of playing. The clarification of the effects of the size and position of tone holes on the fundamental frequency of notes quantitatively will be useful for flute design. Coltman discussed the important acoustical parameters that affect the pitch of the Boehm flute [1]. In Ref. [1], Coltman showed how much the fundamental frequency of notes changes on moving the tone hole $1 \mathrm{~mm}$. Hence, we investigated the effects of the size and position of a tone hole on the fundamental frequency.

The flutes used in this study were constructed in accordance with the model published by Boehm in the 19th century and have 12 or 13 large tone holes and three small tone holes [2]. In the flute design, the size and position of all tone holes are related to the fundamental frequency of the flute. In particular, small tone holes serve as register holes. The small tone hole of the $\mathrm{C}$ key is related to the fundamental frequencies of D6, G\#6, A6, and A\#6, and serves an important role in producing sounds in the high-frequency region of the flute. Although the small tone hole of the $\mathrm{C}$ key is also related to the fundamental frequencies of C\#5, D5, D\#5, and C\#6, this tone hole does not serve as the register hole for these notes. As mentioned above, the $\mathrm{C}$ key has different functions depending on the note. In these two cases above, we consider that changes in the size and position of the tone hole of the $\mathrm{C}$ key also lead to different effects on the fundamental frequency. Hence, we examined the small tone hole of the $\mathrm{C}$ key in this study. In addition, we treated C\#6 and D6 as examples of the two above-described cases, respectively. We did not treat the remaining two small tone holes, called D\# and D trill keys in this study, because these are less frequently used.

In this study, we clarify how changing the size and position of the small tone hole of the $\mathrm{C}$ key affects the fundamental frequencies of two notes, C\#6 and D6, by playing the instrument using an artificial blowing system and through transmission-line matrix modeling to provide aide in the design of the flute. We use an artificial blowing system to eliminate the instability of human blowing and to reduce the calculation cost compared with numerical simulations such as a computational fluid analysis using the finite-element method.

\section{Sound measurement using artificial blowing system}

2.1. Measurement method

We conduct sound measurements using an artificial blowing system to evaluate the effects of the size and position of a tone hole on the fundamental frequency of flutes. We constructed six experimental flutes using the following materials. The head joint of the experimental flutes is plastic and manufactured by Nuvo. The mid- and foot joints of the experimental flutes are made of polyvinyl chloride with an inner diameter of $20 \mathrm{~mm}$.

To investigate the change in fundamental frequency, different sizes and positions of the tone hole were set as follows. We set the sizes of the tone hole of the C key to $8 \mathrm{~mm}$ for an original tone hole and $15.3 \mathrm{~mm}$ for an enlarged tone hole. The sizes of the original and enlarged tone holes of the $\mathrm{C}$ key were based on the sizes of the $\mathrm{C}$ key and a large tone hole of a Nuvo flute, respectively. Although the size of each tone hole of a Nuvo flute differs, we set the same size for three small tone holes and also for 13 large tone holes for simplicity. To compare the effect of tone hole size on the fundamental frequency, we examined two positions of the enlarged tone hole, as shown in Fig. 1.

Figure 2 shows the experimental configuration. The artificial blowing system stores air using a compressor and sends the air to the experimental flute at a constant wind speed monitored with a flow rate meter. We attached a recorder-like mouthpiece to the embouchure hole to blow air into the flute at the same angle. A microphone was installed near the embouchure hole to record the sounds. The parameters of the experiment using the artificial blowing system are the air pressure of $0.6 \times 10^{6} \mathrm{~Pa}$, the flow rate of $25 \mathrm{l} / \mathrm{min}$, the measurement time of $15 \mathrm{~s}$, the number of measurements of 16 and the sampling frequency of $48 \mathrm{kHz}$.

\subsection{Analysis method}

A STFT (short-time Fourier transform) with a window length of 4,096 points and a shift length of 256 points started $3 \mathrm{~s}$ after exceeding $90 \%$ of the peak amplitude, which is the reference level indicating sound to have risen. The duration of the analysis was $10 \mathrm{~s}$ from the start point of STFT. The Hamming window was applied to each frame. The frequency at which the power was maximized by interpolating the peak of the power spectrum of each frame with a quadratic function was taken to be the fundamental frequency.

*e-mail: otakenko@nit.ac.jp

[doi:10.1250/ast.42.282] 


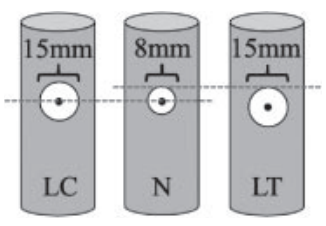

Fig. 1 Dimensions of three types of tone hole of the $\mathrm{C}$ key. "N" is the original tone hole. "LC" is a larger tone hole with its center position aligned with the center of "N." "LT" is a larger tone hole with its top position aligned with the top of "N."

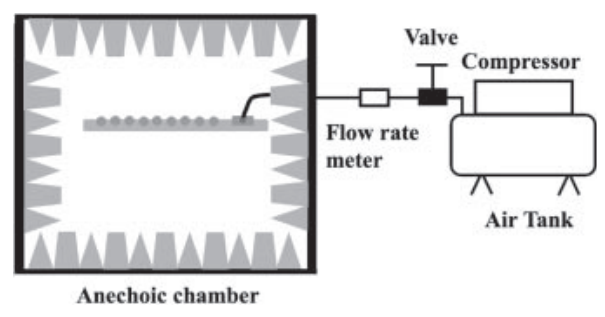

Fig. 2 Experimental configuration.

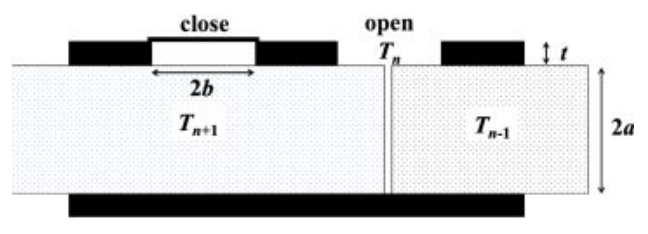

Fig. 3 Dimensions of tone hole and tube.

\section{Resonance frequency obtained using calculation model}

3.1. Two-port matrix model

The input impedance of a flute must be determined to obtain the resonance frequency. We employed the calculation model proposed by Caussé et al. [3]. When a cylindrical tube is composed of $N$ short cylindrical tubes, the sound pressure and volume flow of the input end of a cylindrical tube denoted by $p_{\text {in }}$ and $U_{\text {in }}$, respectively, can be obtained as the product of $N$ transmission matrices of the short cylindrical tubes $T_{n}=\left[\begin{array}{cc}A_{n}(\omega) & B_{n}(\omega) \\ C_{n}(\omega) & D_{n}(\omega)\end{array}\right]$, as shown as

$$
\left[\begin{array}{c}
p_{\text {in }}(\omega) \\
U_{\text {in }}(\omega)
\end{array}\right]=T_{N} T_{N-1} \cdots T_{2} T_{1}\left[\begin{array}{c}
p_{\text {out }}(\omega) \\
U_{\text {out }}(\omega)
\end{array}\right] .
$$

Here, $\omega$ is the angular frequency and $p_{\text {out }}$ and $U_{\text {out }}$ are the sound pressure and volume velocity of the output end, respectively.

We assume that a flute is composed of short cylindrical tubes and open tone holes. Figure 3 shows the dimensions of the tone holes and the tube for the calculation model of the flute. The transmission matrix of a tone hole is inserted between two adjacent transmission matrices of the cylindrical tube. We describe the transmission matrix of a tone hole $T_{\text {hole }}$ and a cylindrical tube $T_{\text {tube }}$ in the following subsection. We set the values of the model parameters of the flute shown in Fig. 3 as follows: length of tube $L=592 \mathrm{~mm}$, inner radius of tube $a=10 \mathrm{~mm}$, radius of large hole $b_{1}=7.65 \mathrm{~mm}$, radius of small hole $b_{\mathrm{s}}=4 \mathrm{~mm}$, thickness of tube wall $t=3 \mathrm{~mm}$, radius of curvature at either end of hole $r_{\mathrm{c}}=0.3 \mathrm{~mm}$, and area of embouchure hole $A_{\mathrm{em}}=94.2 \mathrm{~mm}^{2}$. The area of the embouchure hole was approximated from that of an ellipse with a semi-major axis of $6 \mathrm{~mm}$ and a semi-minor axis of $5 \mathrm{~mm}$. The inner radius of the actual flute is $9.5 \mathrm{~mm}$, but we set it to $10 \mathrm{~mm}$ on the basis of the measurement result of our experimental flute made of polyvinyl chloride. We measured the inner radius using calipers.

We set the values of various parameters in air as follows [4]: sound velocity $c=346.3 \mathrm{~m} / \mathrm{s}$, density $\rho=1.17 \mathrm{~kg} / \mathrm{m}^{3}$, viscosity $\mu=1.8 \times 10^{-5} \mathrm{~kg} /(\mathrm{m} \cdot \mathrm{s})$, thermal conductivity $\lambda=$ $6.2 \times 10^{-3} \mathrm{cal} /(\mathrm{m} \cdot \mathrm{s} \cdot \mathrm{K})$ when the temperature is $25^{\circ} \mathrm{C}$, specific heat at constant pressure $C_{\mathrm{p}}=240 \mathrm{cal} /(\mathrm{kg} \cdot \mathrm{K})$, and specific heat ratio $\gamma=1.4$.

3.2. Transmission matrix of tone hole

The transmission matrix of a tone hole $T_{\text {hole }}$ can be represented by the shunt impedance $Z_{\mathrm{s}}$ and the series impedance $Z_{\mathrm{a}}$ as follows [5]:

$$
T_{\text {hole }}(\omega) \approx\left[\begin{array}{cc}
1 & Z_{\mathrm{a}}(\omega) \\
\frac{1}{Z_{\mathrm{S}}(\omega)} & 1
\end{array}\right] .
$$

The shunt impedance can be represented by [6-8]

$$
Z_{\mathrm{s}}(\omega)=\frac{\rho c}{\pi b^{2}}\left(i k t_{\mathrm{e}}+\xi\right),
$$

where $k(=\omega / c)$ is the wave number, $c$ is the sound velocity, and $b$ is the radius of the tone hole. The effective length $t_{\mathrm{e}}$ can be represented by the sum of the thickness of the tube wall $t$ and the three corrections:

$$
t_{\mathrm{e}}=t+t_{\mathrm{m}}+t_{\mathrm{i}}+t_{\mathrm{r}} .
$$

The three corrections are based on Ref. [5]. The series impedance $Z_{\mathrm{a}}$ is given by

$$
Z_{\mathrm{a}}=i \frac{\rho c}{\pi b^{2}} k t_{\mathrm{a}}
$$

where the effective length $t_{\mathrm{a}}$ is

$$
t_{\mathrm{a}}=-b \delta^{2}\{0.35-0.06 \tanh (2.7 t / b)\} .
$$

\subsection{Transmission matrix of cylindrical tube}

We modeled the transmission matrix of a cylindrical tube $T_{\text {tube }}$ by considering the visco-thermal loss [3]. The transmission matrix of a short cylindrical tube is expressed as

$$
T_{\text {tube }}=\left[\begin{array}{cc}
\cosh \left(\Gamma L_{n}\right) & \frac{\zeta}{A} \sinh \left(\Gamma L_{n}\right) \\
\frac{A}{\zeta} \sinh \left(\Gamma L_{n}\right) & \cosh \left(\Gamma L_{n}\right)
\end{array}\right],
$$

where $A$ is the area of the bore of the tube and $L_{n}$ is the length of the $n$-th short cylindrical tube. Here, the propagation constant $\Gamma$ and the wave impedance $\zeta$ are given by

$$
\Gamma=\sqrt{Z_{\mathrm{v}} Y_{\mathrm{t}}}, \quad \zeta=\sqrt{\frac{Z_{\mathrm{v}}}{Y_{\mathrm{t}}}} .
$$

The series impedance per unit length $Z_{\mathrm{v}}$ and the shunt admittance per unit length $Y_{\mathrm{t}}$ are modeled with the following equations: 


$$
\begin{aligned}
& Z_{\mathrm{v}}=i \omega \rho\left(1+\frac{2}{r_{\mathrm{v}}}(1-i)-\frac{3 i}{r_{\mathrm{v}}^{2}}\right), \\
& Y_{\mathrm{t}}=\frac{i \omega}{\rho c^{2}}\left[1+(\gamma-1)\left(\frac{\sqrt{2}}{r_{\mathrm{t}}}(1-i)+\frac{i}{r_{\mathrm{t}}^{2}}\right)\right] .
\end{aligned}
$$

Here, $r_{\mathrm{v}}=a \sqrt{\omega \rho / \mu}$ and $r_{\mathrm{t}}=a \sqrt{\omega \rho C_{\mathrm{p}} / \lambda}$ [4]. In Ref. [4], the pipe radius $a$ was regarded as the arithmetic mean of the radii of the two ends. Hence, in cylindrical sections, we treated both ends of the tube as having the same radius.

3.4. Input impedance of flute

The input impedance of the flute can be represented by the following equation [4]:

$$
Z_{\text {in }}(\omega)=\frac{p_{\text {in }}(\omega)}{U_{\text {in }}(\omega)}=\frac{A(\omega) Z_{\mathrm{r}}(\omega)+B(\omega)}{C(\omega) Z_{\mathrm{r}}(\omega)+D(\omega)} .
$$

Here, $Z_{\mathrm{r}}(\omega)$ is the radiation impedance that can be modeled using the radiation from a round piston head surrounded by an infinite baffle. $Z_{\mathrm{r}}(\omega)$ is approximated as

$$
Z_{\mathrm{r}}(\omega)=\frac{p_{\text {out }}(\omega)}{U_{\text {out }}(\omega)}=\frac{\zeta}{A} \frac{i \omega R_{\mathrm{r}} L_{\mathrm{r}}}{R_{\mathrm{r}}+i \omega L_{\mathrm{r}}},
$$

where

$$
R_{\mathrm{r}}=\frac{128}{9 \pi^{2}}, \quad L_{\mathrm{r}}=\frac{8 a}{3 \pi c} .
$$

\subsection{Calculation of input impedance}

We assume that a flute is composed of cylindrical tubes and open tone holes. Figure 4 shows an example of the dimensions of the flute and the fingering patterns of notes C\#6 and D6. The size and position of the tone hole of the $\mathrm{C}$ key shown in Fig. 4 correspond to "N" shown in Fig. 1. Although the actual length of the tube is $592 \mathrm{~mm}$, to conduct a simulation, we should consider the end correction produced at the embouchure hole. The end correction produced at the embouchure hole has been defined as [9]

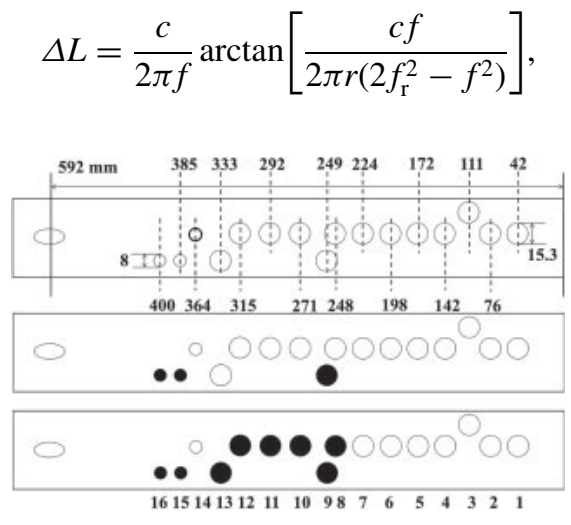

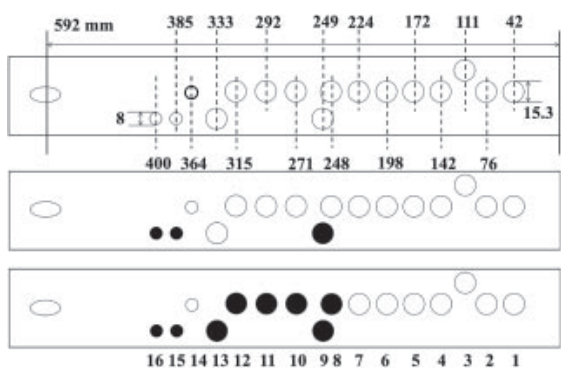

Fig. 4 Position of tone holes (top) and fingerings for notes C\#6 (middle) and D6 (bottom). The top figure shows the distance from the output end of the tube to the center of the tone holes and their sizes. In addition, $\bigcirc$ and $\bigcirc$ denote open and closed tone holes, respectively. The bottom figure shows the tone hole numbers. Tone hole No. 14 corresponds to the tone hole of the $\mathrm{C}$ key in this study. The sizes of tone holes Nos. 1-13 are the same. In addition, the sizes of tone hole Nos. 14-16 are the same. where $r$ is the length between the center of the embouchure hole and the cork, and $f_{\mathrm{r}}$ is the Helmholtz resonator frequency. The length $r$ is typically set to $17 \mathrm{~mm}$ for the Boehm flute and the frequency $f_{17}$ is approximately $1,450 \mathrm{~Hz}$ for the normal playing condition [9]. When $r$ is $17 \mathrm{~mm}$ and $f_{17}$ is $1,450 \mathrm{~Hz}, \Delta L$ is approximately $42 \mathrm{~mm}$. If the player sufficiently covers the embouchure hole, $f_{\mathrm{r}}$ decreases. Hence, we also consider the following conditions: $r=17 \mathrm{~mm}$ and $f_{17}=1,200 \mathrm{~Hz}$. Under these conditions, $\Delta L$ is approximately $58 \mathrm{~mm}$. We inserted a 42 or $58 \mathrm{~mm}$ cylindrical tube as the end correction between the embouchure hole and tone hole No. 16 shown in Fig. 4.

\section{Result}

4.1. Fundamental frequency obtained experimentally

In the case of the experimental flutes for $\mathrm{C} \# 6$, the average fundamental frequencies were $1,120.3 \mathrm{~Hz}$ for $\mathrm{N}, 1,154.3 \mathrm{~Hz}$ for $\mathrm{LC}$, and $1,142.1 \mathrm{~Hz}$ for LT. If the size of the $\mathrm{C}$ key is set to $15.3 \mathrm{~mm}$, the fundamental frequency will be $30 \mathrm{~Hz}$ higher for LC and $20 \mathrm{~Hz}$ higher for LT compared with the fundamental frequency of $\mathrm{N}$. It was observed that the fundamental frequency decreased from LC to LT, because the position of the tone hole of the $\mathrm{C}$ key for LT was closer to the output end of the tube than that for LC, and the effective length of LT became larger than that of LC.

In the case of the experimental flutes for D6, the average fundamental frequencies were $1,161.3 \mathrm{~Hz}$ for $\mathrm{N}, 1,169.0 \mathrm{~Hz}$ for LC, and $1,170.0 \mathrm{~Hz}$ for LT. When the size of the C key tone hole was set to $15.3 \mathrm{~mm}$, the fundamental frequency increased by approximately $5 \mathrm{~Hz}$ for both LC and LT compared with that of $\mathrm{N}$, and there was no significant change in fundamental frequency regardless of the tone hole conditions.

4.2. Resonance frequency obtained using calculation model

The resonance frequency corresponds to the admittance peak frequency. The admittance is defined as the reciprocal of the input impedance. Each peak of the admittance represents a resonance mode of the flutes. Table 1 shows the resonance frequencies of the experimental flutes for C\#6 and D6 when $\Delta L=42$ and $58 \mathrm{~mm}$.

We focused on the resonance frequencies of the $f_{2}$ mode of the experimental flutes for $\mathrm{C} \# 6$, and the $f_{3}$ mode of the experimental flutes for D6. A comparison of the tone hole conditions $\mathrm{N}$ and $\mathrm{LC}$ showed that the relative error of the resonance frequency of C\#6 was approximately $5 \%$ for $\Delta L=$

Table 1 Resonance frequency $f_{n}$ obtained using calculation models of the experimental flutes. $\Delta L=42 \mathrm{~mm}$ (left) and $58 \mathrm{~mm}$ (right).

\begin{tabular}{rrrrrr}
\hline & & $f_{2}$ & \multicolumn{1}{c}{$f_{3}$} & \multicolumn{1}{c}{$f_{2}$} & $f_{3}[\mathrm{~Hz}]$ \\
\hline \multirow{4}{*}{ C\#6 } & $\mathrm{N}$ & 1,169 & 1,736 & 1,109 & 1,651 \\
& $\mathrm{LC}$ & 1,216 & 1,815 & 1,151 & 1,720 \\
& $\mathrm{LT}$ & 1,203 & 1,798 & 1,140 & 1,704 \\
\hline \multirow{4}{*}{ D6 } & $\mathrm{N}$ & 909 & 1,231 & 890 & 1,183 \\
& $\mathrm{LC}$ & 1,006 & 1,243 & 993 & 1,188 \\
& $\mathrm{LT}$ & 1,020 & 1,236 & 1,006 & 1,184 \\
\hline
\end{tabular}


42 and $58 \mathrm{~mm}$. In contrast, the relative error of the resonance frequency of D6 was below $1 \%$ for $\Delta L=42$ and $58 \mathrm{~mm}$. Here, the relative error was calculated using the ratio of the difference between the resonance frequencies for $\mathrm{LC}$ and $\mathrm{N}$ to the resonance frequency for $\mathrm{N}$. Although the resonance frequency was a semitone higher than the actual fundamental frequencies of C\#6 and D6 when $\Delta L$ was set assuming a normal playing condition, the tendency for the resonance frequency to change depending on the tone hole conditions was the same for $\Delta L=42$ and $58 \mathrm{~mm}$.

\section{Discussion}

We investigate the effects of the size and position of the tone hole of the $\mathrm{C}$ key on the fundamental frequency by comparing $\mathrm{N}$ with LC and LT. For the note C\#6, the resonance frequency of the $f_{2}$ mode of LC was the highest. In addition, for the note D6, the difference between the resonance frequencies of the $f_{3}$ mode was approximately $5 \mathrm{~Hz}$ at maximum, as shown in Table 1 . These results are in good agreement with the measurement results. That is, we confirmed that the effects of the size and position of the tone holes differed depending on the note. The reason why the effects were different is that the function of the tone hole of the $\mathrm{C}$ key differs between $\mathrm{C} \# 6$ and D6. For note C\#6, the tone hole of the $\mathrm{C}$ key is the first open tone hole, as shown in Fig. 4, and much radiation occurs from the tone hole of the $\mathrm{C}$ key. Hence, the size and position of the tone hole of the $\mathrm{C}$ key affect the resonance frequency of $\mathrm{C \# 6}$. On the other hand, for note D6, the tone hole of the C key is allocated at about twothirds of the effective length and acts as a register hole when the fingering is as shown in [10]. A pressure node occurs near the tone hole of the $\mathrm{C}$ key and less radiation occurs from the tone hole of the $\mathrm{C}$ key. Hence, the effects of the size and position of the tone hole of the $\mathrm{C}$ key on the resonance frequency of the $f_{3}$ mode of D6 are small.

On the basis of the above results, to investigate the effects of the size and position of the tone hole in more detail using the calculation model, the resonance frequency was calculated when the position of the $C$ key tone hole was moved at $1 \mathrm{~mm}$ increments. The calculation results are shown in Fig. 5. The resonance frequencies of the $f_{2}$ mode for $\mathrm{C} \# 6$ and the $f_{3}$ mode for D6 are shown. In this figure, it is seen that the resonance frequency for $\mathrm{C} \# 6$ changed linearly with the size and position of the tone hole of the $\mathrm{C}$ key. In contrast, the resonance frequency of the $f_{3}$ mode for D6 showed a nonlinear relationship with by the position of the tone hole of the $\mathrm{C}$ key with a larger tone hole.

\section{Conclusion}

Although the results of this study are limited to $\mathrm{C} \# 6$ and D6, we confirmed the effects of the size and position of tone holes on the fundamental frequency of these notes quantitatively. These results indicate that it is possible to change the

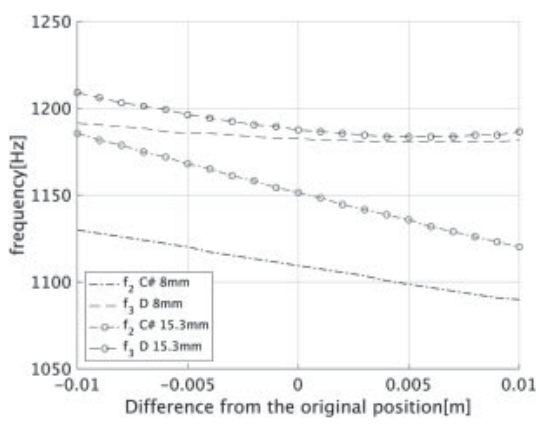

Fig. 5 Resonance frequency when the position of the $\mathrm{C}$ key tone hole is moved in $1 \mathrm{~mm}$ increments. The $f_{2}$ mode for $\mathrm{C} \# 6$ and the $f_{3}$ mode for D6 are shown. The sizes of tone holes are $8 \mathrm{~mm}$ and $15.3 \mathrm{~mm}$. The positive difference from the original position indicates the $\mathrm{C}$ key tone hole is away from the embouchure hole.

fundamental frequency of $\mathrm{C} \# 6$ by changing the size and position of the tone hole of the $\mathrm{C}$ key within a certain range without significantly affecting the fundamental frequency of D6.

By accumulating experimental results, we consider that these findings will contribute to further development of flutes. Hence, we will continue to construct experimental flutes with different sizes and positions of tone holes, and investigate how changing the size and position of the tone holes affects the fundamental frequency.

\section{References}

[1] J. W. Coltman, "Designing the scale of the Boehm flute," Woodwind Q., Issue 4, pp. 24-41 (1994).

[2] T. Boehm, The Flute and Flute-playing, translated by D. C. Miller (Dover, New York, 1964).

[3] R. Caussé, J. Kergomard and X. Lurton, "Input impedance of brass musical instruments: Comparison between experiment and numerical models," J. Acoust. Soc. Am., 75, 241-254 (1984).

[4] S. Adachi and M. Yamada, "An acoustical study of sound production in biphonic singing, Xöömij," J. Acoust. Soc. Am., 105, 2920-2932 (1999).

[5] S. Adachi, "Resonance modes of a flute with one open tone hole," Acoust. Sci. \& Tech., 38, 14-22 (2017).

[6] D. H. Keefe, "Theory of the single woodwind tone hole," $J$. Acoust. Soc. Am., 72, 676-687 (1982).

[7] D. H. Keefe, "Experiments on the single woodwind tone hole," J. Acoust. Soc. Am., 72, 688-699 (1982).

[8] D. H. Keefe, "Woodwind air column models," J. Acoust. Soc. Am., 88, 35-51 (1990).

[9] A. H. Benade and J. W. French, "Analysis of the flute head joint,” J. Acoust. Soc. Am., 37, 679-691 (1965).

[10] J. Wofle, J. Smith, J. Tann and N. H. Fletcher, "Acoustic impedance spectra of classical and modern flutes," J. Sound Vib., 243, 127-144 (2001). 